\title{
Depression and Perceived Stress, but Not Anxiety, are Associated with Elevated Inflammation in an Obese Adult Population
}

This article was published in the following Dove Press journal: Risk Management and Healthcare Policy

\section{Bin Zou' \\ Chenfang Miao $^{2}$ \\ Jiliang Chen'}

'Department of Orthopaedics, Affiliated Mindong Hospital of Fujian Medical University, Fuan City, Fu Jian 355000, People's Republic of China; ${ }^{2}$ Department of Anesthesiology, Affiliated Mindong Hospital of Fujian Medical University, Fuan City, Fu Jian 355000, People's Republic of China
Correspondence: Jiliang Chen Department of Orthopaedics, Affiliated Mindong Hospital of Fujian Medical University, No. 89 Heshan Road, Fuan City 355000, Fujian Province,

People's Republic of China

$\mathrm{Tel}+8613959325972$

Email fujiancjl@I63.com
Background: Anxiety, depression and perceived stress are risk factors for adverse health problems. Inflammation participates in the development of chronic diseases such as psychiatric disorders. This study explored the relationships between inflammatory biomarkers and depression, anxiety and perceived stress in an obese adult population.

Methods: The relationships between psychological scores and inflammatory markers were analyzed.

Results: A higher BMI was not correlated with a higher anxiety score $(\mathrm{P}=0.152)$; however, BMI was positively associated with a higher depression score $(\mathrm{P}<0.001)$ and a higher perceived stress score $(\mathrm{P}<0.001)$. Multivariate linear regression analysis revealed that in participants with $\mathrm{BMI} \geq 30$ and $25 \leq \mathrm{BMI}<30$, depression and perceived stress were significantly and independently associated with ICAM-1, E-selectin and CRP, but these associations were not observed in participants with $\mathrm{BMI}<25$. The anxiety score was not associated with any inflammatory marker in any group of subjects, as determined by multivariate analysis.

Conclusion: Depression and perceived stress were strongly associated with increased serum levels of pro-inflammatory markers, including ICAM-1, E-selectin and CRP, among a general obese population from the United States. These results further suggest that depression and perceived stress might also be chronic systemic inflammatory diseases.

Keywords: inflammatory markers, anxiety, depression, perceived stress, obesity

\section{Introduction}

Psychiatric disorders, such as anxiety and depression, are established risk factors for health problems such as cardiovascular diseases (CVDs). ${ }^{1-4}$ Many previous studies have shown significant associations between CVD risk and anxiety or depression, which may be explained in part by increasing levels of circulating pro-inflammatory markers, including interleukin-6 (IL-6) and tumor necrosis factor-a (TNF-a). ${ }^{5-9}$ It is very important for us to understand the increasing levels of inflammation biomarkers in circulating blood because chronic elevation of the serum levels of inflammation biomarkers such as C-reactive protein (CRP) have been shown to predict CVDs, including coronary heart disease, stroke and others, in many clinical studies. ${ }^{10,11}$ However, studies on the relationships between psychiatric disorders, such as anxiety and depression, and serum inflammation levels are conflicting and few.

Circulating inflammation biomarker levels are also elevated in individuals with obesity, ${ }^{12}$ a known risk factor for CVDs. Importantly, obese people are more prone 
to depression and anxiety, particularly individuals with severe obesity. ${ }^{3,13}$ Among people who have had weight loss surgery, the lifetime prevalence of anxiety or depression is also higher. ${ }^{14,15}$ Considering the high rate of depression or anxiety in persons with obesity, focusing on whether depression and anxiety are related to inflammation in obese populations is clinically important. The stress response can be considered the formation of adaptation. $^{16,17}$ Some animal studies have also shown that chronically stressed individuals have higher proinflammatory levels that result from glucocorticoid resistance. $^{18,19}$ An overreaction to stress is linked with increased inflammatory responses and may impair immune function, which may aging. ${ }^{20}$ Previous studies have also demonstrated that the level of inflammation in mice with high stress increased significantly. ${ }^{20,21}$ Furthermore, it has been confirmed that perceived stress is closely related to the occurrence and prognosis of CVDs. ${ }^{22-25}$

Considering the potential link between psychological problems and systemic inflammation, we aimed to assess the association between depression, anxiety and perceived stress scores and inflammation biomarkers in a cross-sectional study with obese and nonobese subjects at high risk of adverse health outcomes. This work reports a multicenter, large-scale cross-sectional study with sufficient inflammatory markers for analysis, which can further clarify the associations of anxiety, depression and perceived stress scores with systemic inflammatory levels in an obese population.

\section{Materials and Methods Study Population}

The data from the Midlife in the United States (MIDUS) study were analyzed. The MIDUS study assesses the correlation between various sociological factors and mental health. As a subpart of the MIDUS study, 1255 subjects finished the Biomarkers Project between 2004 and 2009. These subjects had comprehensive biological assessments that were integrated with sociological factors. ${ }^{26}$ Detailed information regarding the Biomarkers Project in the MIDUS study is available elsewhere. ${ }^{26,27}$ The complete data and specific codebooks are also available at http:// www.midus.wisc.edu/. The Biomarkers Project data were obtained from the University of California-Los Angeles, Georgetown University and the University of WisconsinMadison between 2004 and 2009. All subjects completed blood collection and analysis, a detailed medical history interview and a self-administered questionnaire during a 2day visit to one of the three locations. The traveling expenses of each subject were covered, and the subjects were additionally remunerated $\$ 200$ for participating in the study.

Blood samples from all subjects were obtained and analyzed during the 2-day visit. Inflammatory biomarker measurements were performed by a fasting blood draw. The following inflammatory biomarkers were measured: CRP, IL-6, interleukin-8 (IL-8), interleukin-10 (IL-10), TNF-a, E-selectin and intracellular adhesion molecule-1 (ICAM-1). Subjects with a BMI $<25$ were defined as the normal-weight group, and subjects with a BMI $\geq 30$ were defined as the obese group. Subjects with $25 \leq \mathrm{BMI}<30$ were classified as the overweight group. The number of individuals with a low BMI (BMI <18) was not sufficient for a separate analysis; therefore, they were excluded from the normal-weight group. The MIDUS study was approved by the Ethics Committees of the University of California-Los Angeles, Georgetown University and the University of Wisconsin-Madison, and all participants gave written informed consent. Most of the TILDA data were obtained through the data-sharing platform available for free use by researchers worldwide. According to the Declaration of Helsinki guidelines, the Ethics Committee of The Affiliated Mindong Hospital of Fujian Medical University approved this study.

\section{Anxiety, Depression and Perceived Stress Scores}

The Hospital Anxiety and Depression Scale-Anxiety Subscale (HADS-A) is a common method for assessing the severity of anxiety in general populations. ${ }^{28}$ The examination includes seven items. All subjects were assessed by each item with 4 grades ( $0-3$ points) for the past month. A higher anxiety score suggests more severe anxiety symptoms. The Centre for Epidemiological Studies Depression (CES-D) scale can be used to assess depressive symptoms and has been used widely in different populations. ${ }^{29} \mathrm{~A}$ higher score usually indicates more severe depressive symptoms. The Perceived Stress Scale (PSS) is a 10-item measure that can assess the perceived degree of stress in the lives of the subjects. ${ }^{30}$ Individual scores can range from 10 to 50, with a higher score indicating higher perceived stress. 


\section{Covariates}

Some confounding factors are associated with anxiety, depression, perceived stress and inflammation. Sociodemographic and other data, such as age, gender, race, education, smoking status, marital status, alcohol consumption and medications, were collected from self-administered questionnaires between 2004 and 2009. BMI was objectively measured from the inperson visit. A detailed description of these covariates has been described elsewhere. ${ }^{26,27}$

\section{Statistical Analysis}

The analyses were performed using SPSS24 (SPSS Inc., IL, USA). $P \leq 0.05$ was considered statistically significant. The Kolmogorov-Smirnov test was used to assess the normality of the data. None of the continuous variables in this study were normally distributed. The correlations between BMI and anxiety, depression and perceived stress scores were performed by Spearman's analysis. All variables that were standardized with z-scores were evaluated by Pearson's correlation coefficient, which aimed to assess the associations between serum inflammatory biomarkers and anxiety, depression and perceived stress scores. Multivariate linear regression analysis was performed to assess the associations between serum inflammatory biomarkers and anxiety, depression and perceived stress scores. Finally, an additional stratified analysis by the BMI value was performed to assess the associations between psychological scores and inflammatory biomarkers.

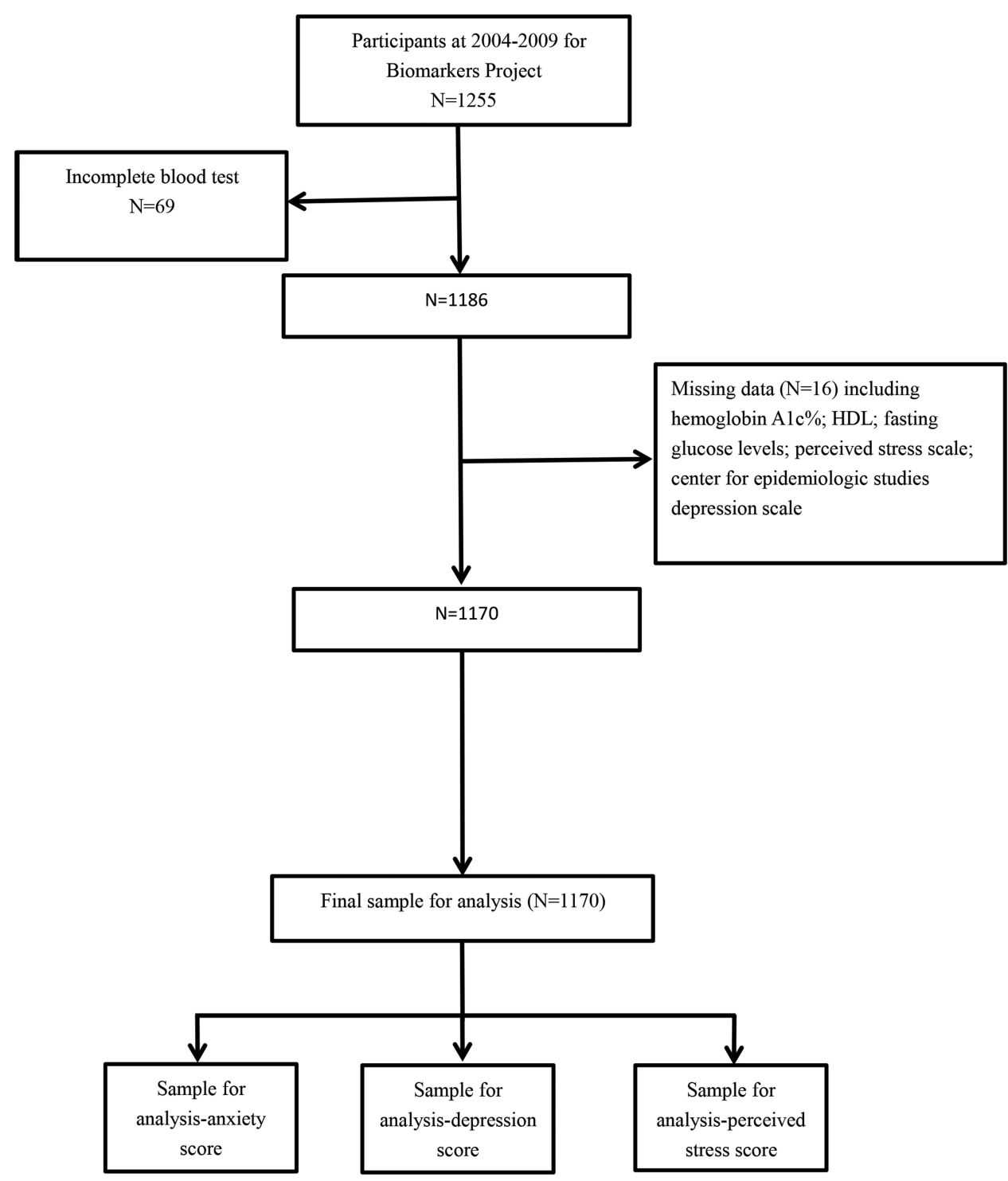

Figure I A detailed flow chart of describing the participants included in this study. 


\section{Results}

\section{The Characteristics of the Study Subjects}

In total, 1255 participants completed the Biomarkers Project between 2004 and 2009. Of these individuals, 1170 were included in the final sample for analysis (Figure 1). The characteristics of all included subjects were as follows (Table 1): 505 participants (43.16\%) were male, 1106 (94.53\%) were white, 451 (38.55\%) had a bachelor's degree or higher, 250 (21.37\%) were currently married, 171 (14.62\%) were current smokers, and 21 (1.79\%) were current drinkers. The median age of all subjects was 54 years. BMI was not associated with the anxiety score $(r=-0.056, \mathrm{P}=0.152$, Figure 2A). However, BMI was positively associated with the depression score $(r=0.168, \mathrm{P}<0.001$, Figure $2 \mathrm{~B})$ and the perceived stress score $(r=0.167, \mathrm{P}<0.001$, Figure $2 \mathrm{C})$.

\section{Relationships Between Inflammation} Biomarkers and Anxiety, Depression and Perceived Stress Scores by Pearson

\section{Correlation Analysis}

The depression score was positively related to the levels of serum inflammation markers, including E-selectin $(\mathrm{S} \beta=0.112$, $\mathrm{P}<0.001)$, CRP $(\mathrm{S} \beta=0.109, \mathrm{P}<0.001)$ and ICAM-1 $(\mathrm{S} \beta=0.098$, $\mathrm{P}=<0.001)$. The perceived stress score was positively related to the levels of serum inflammation markers, including E-selectin $(\mathrm{S} \beta=0.094, \quad \mathrm{P}=0.001)$ and $\mathrm{CRP} \quad(\mathrm{S} \beta=0.080$, $\mathrm{P}=0.006$ ). Conversely, the anxiety score was not associated with any serum inflammation marker levels in this study (Table 2).

\section{Relationships Between Inflammation} Biomarkers and Anxiety, Depression and Perceived Stress Scores After Adjusting for Relevant Confounding Factors

To further exclude the influence of related confounding factors, the correlations between serum inflammatory biomarkers and anxiety, depression and perceived stress scores were performed by multiple linear regression analysis (Table 3 ). The depression score was positively related to E-selectin $(\mathrm{S} \beta=0.049, \mathrm{P}<0.001)$, ICAM-1 $(\mathrm{S} \beta=0.064$, $\mathrm{P}<0.001)$ and $\mathrm{CRP}(\mathrm{S} \beta=0.068, \mathrm{P}<0.001)$ after adjustment was made for age, gender, race, marital status, education, smoking status, alcohol consumption, BP and medications (Model 3). The perceived stress scores were also positively associated with ICAM-1 $(\mathrm{S} \beta=0.017, \mathrm{P}=0.022)$, E-selectin $(\mathrm{S} \beta=0.040, \mathrm{P}=0.014)$ and $\mathrm{CRP} \quad(\mathrm{S} \beta=0.067$,
Table I Participants' Characteristics

\begin{tabular}{|c|c|}
\hline Variables & M (IQR) or N (\%) \\
\hline Age (years) & $54.00(45.00-63.00)$ \\
\hline Gender (male), n (\%) & 505 (43.16\%) \\
\hline Race (white), n (\%) & $1106(94.53 \%)$ \\
\hline Education (with bachelor's degree or higher), $\mathrm{n}(\%)$ & $451(38.55 \%)$ \\
\hline BMI & $28.50(25.13-32.85)$ \\
\hline Currently married, n (\%) & $250(21.37 \%)$ \\
\hline Current smoker, n (\%) & $17 \mid(\mid 4.62 \%)$ \\
\hline Current drinker, n (\%) & $21(1.79 \%)$ \\
\hline Systolic BP (mmHg) & $130.00(\mid 19.00-143.00)$ \\
\hline Diastolic BP (mmHg) & $75.00(68.00-82.00)$ \\
\hline Anxiety scores & $1.80(1.40-2.20)$ \\
\hline Depression scores & $6.00(3.00-12.00)$ \\
\hline Perceived stress scores & $22.00(18.00-26.00)$ \\
\hline \multicolumn{2}{|l|}{ Medications } \\
\hline Antidepressants, n (\%) & $161(13.76 \%)$ \\
\hline Antihypertension, n (\%) & $434(37.09 \%)$ \\
\hline Antihyperlipidemic agents, n (\%) & $348(29.74 \%)$ \\
\hline ACEI, $n(\%)$ & $113(9.66 \%)$ \\
\hline Beta adrenergic blocking agents, $\mathrm{n}(\%)$ & $159(13.59 \%)$ \\
\hline Calcium channel blocker, n (\%) & $97(8.29 \%)$ \\
\hline Diuretics, n (\%) & $117(10.00 \%)$ \\
\hline \multicolumn{2}{|l|}{ Blood samples } \\
\hline Total cholesterol (mg/dL) & $183.00(159.00-211.00)$ \\
\hline Triglycerides (mg/dL) & $106.00(77.00-154.00)$ \\
\hline HDL cholesterol (mg/dL) & $53.00(43.00-66.00)$ \\
\hline LDL cholesterol (mg/dL) & $101.00(80.00-127.25)$ \\
\hline Fasting glucose levels (mg/dL) & $96.00(90.00-105.00)$ \\
\hline Hemoglobin Alc \% & $5.82(5.60-6.20)$ \\
\hline Fasting insulin levels (ulU/mL) & $10.00(6.00-16.00)$ \\
\hline HOMA-IR & $2.39(\mid .42-4.31)$ \\
\hline Serum IL6 (pg/mL) & $0.80(0.56-1.21)$ \\
\hline Serum IL8 (pg/mL) & $12.36(9.13-15.76)$ \\
\hline Serum ILIO (pg/mL) & $0.22(0.16-0.32)$ \\
\hline Serum TNF-a (pg/mL) & $2.05(1.69-2.50)$ \\
\hline C-reactive protein (ug/mL) & $1.43(0.69-3.63)$ \\
\hline Serum E-selectin $(\mathrm{ng} / \mathrm{mL})$ & $38.83(28.03-51.73)$ \\
\hline Serum ICAM-I (ng/mL) & $272.11(217.72-334.47)$ \\
\hline
\end{tabular}

Abbreviations: BMI, body mass index; BP, blood pressure; $\mathrm{ACEl}$, angiotensinconverting enzyme inhibitors; HDL, high-density lipoprotein; LDL, low-density lipoprotein; HOMA-IR, homeostasis model assessment of insulin resistance; IL, interleukin; TNF-a, tumor necrosis factor-a; ICAM-I, intercellular adhesion molecule-I.

$\mathrm{P}=0.009$ ) after adjustment of these confounding factors. The anxiety scores were not associated with the serum levels of any inflammatory biomarker in our study, similar to the results of the univariate analysis.

Existing studies have shown a strong link between obesity and inflammation levels in vivo and in vitro. ${ }^{31,32}$ Therefore, stratified BMI (three groups) was analyzed to assess whether inflammatory biomarker levels are related to these scores independent of BMI in subjects with obesity. As 
A

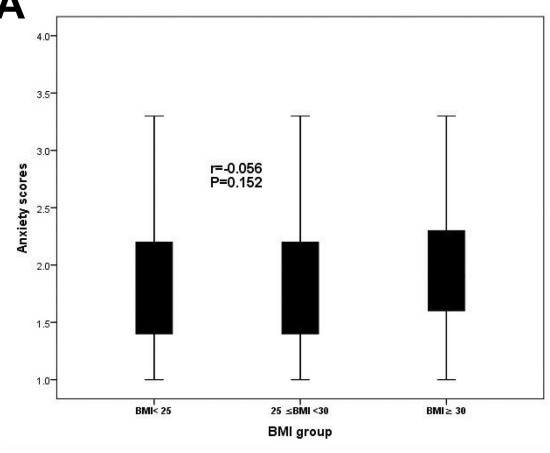

B

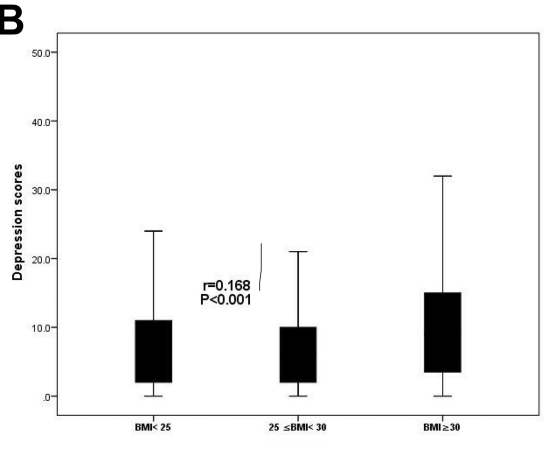

c

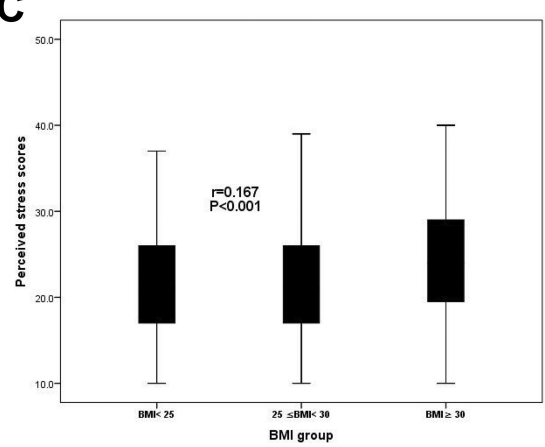

Figure 2 (A) The relationship between BMI and anxiety scores. (B) The relationship between BMI and depression scores. (C) The relationship between BMI and perceived stress scores.

shown in Table 4, in the normal-weight group with $\mathrm{BMI}<25$, anxiety, depression and perceived stress scores had no correlations with the serum levels of any inflammation biomarker (all $\mathrm{P}>0.05$ ). However, in the obese group with $\mathrm{BMI} \geq 30$ and overweight group with $25 \leq \mathrm{BMI}<30$, depression and the perceived stress scores remained associated with ICAM-1, E-selectin, and CRP (all P>0.05). Similarly, the anxiety scores remained unrelated to any of the assessed inflammatory markers in the three groups. These findings suggest that depression and perceived stress, but not anxiety, are associated with elevated inflammation in overweight and obese subjects but not in normal-weight subjects.

\section{Discussion}

This study aimed to assess the relationships between depression, anxiety and perceived stress scores and inflammatory biomarkers that can predict obesity-related diseases in an obese adult population. In addition to the anxiety score, univariate analysis suggested that depression and perceived stress scores were positively related to inflammatory factors. Furthermore, upon multivariate analysis, depression and perceived stress scores remained associated with higher serum levels of inflammatory biomarkers after adjusting for confounding factors. Our findings contribute to the existing literature in many aspects. First, the MIDUS study is a longitudinal study with large samples, which provided sufficient inflammatory markers to analyze. Therefore, the current work expands upon previous studies on the association of psychological scores with inflammation in adult populations. Second, our multivariate and stratified analysis findings demonstrated that depression and perceived stress scores were independently and significantly related to the serum levels of ICAM-1and E-selectin in obese adults but not in nonobese adults. Inflammation is closely related to the occurrence and prognosis of chronic diseases ${ }^{10,11}$ and may provide a pathway linking depression and perceived stress to an increased risk of adverse health outcomes. Additionally, the levels of serum TNF-a IL-10, IL-8 and IL-6, which are reliable and comprehensive markers, were measured in our study

Table 2 Univariate Analysis of Relationships Between Inflammation and Anxiety, Depression and Perceived Stress Scores

\begin{tabular}{|l|l|l|l|l|l|l|}
\hline \multirow{2}{*}{ Variables } & \multicolumn{2}{l|}{ Anxiety Scores } & \multicolumn{2}{l|}{ Depression Scores } & \multicolumn{2}{l|}{ Perceived Stress Scores } \\
\cline { 2 - 7 } & $\mathbf{S} \boldsymbol{\beta}$ & $\boldsymbol{P}$ value & $\mathbf{S} \boldsymbol{\beta}$ & $\boldsymbol{P}$ value & S $\boldsymbol{l}$ & $\boldsymbol{P}$ value \\
\hline Serum IL6 $(\mathrm{pg} / \mathrm{mL})$ & 0.009 & 0.747 & 0.039 & 0.187 & 0.050 & 0.057 \\
Serum IL8 $(\mathrm{pg} / \mathrm{mL})$ & -0.023 & 0.434 & 0028 & 0.330 & -0.025 & 0.396 \\
Serum ILIO $(\mathrm{pg} / \mathrm{mL})$ & -0.008 & 0.773 & 0.002 & 0.953 & -0.020 & 0.505 \\
Serum TNF-a $(\mathrm{pg} / \mathrm{mL})$ & -0.009 & 0.771 & 0.028 & 0.333 & -0.032 & 0.276 \\
C-reactive protein $(\mathrm{ug} / \mathrm{mL})$ & 0.004 & 0.894 & 0.109 & $<0.001$ & 0.080 & 0.006 \\
Serum E-selectin $(\mathrm{ng} / \mathrm{mL})$ & 0.038 & 0.199 & 0.112 & $<0.001$ & 0.094 & 0.001 \\
Serum ICAM-I $(\mathrm{ng} / \mathrm{mL})$ & 0.030 & 0.309 & 0.098 & $<0.001$ & 0.032 & 0.270 \\
\hline
\end{tabular}

Note: Pearson correlation analysis was used.

Abbreviations: IL, interleukin; TNF-a, tumor necrosis factor-a; ICAM-I, intercellular adhesion molecule-I. 


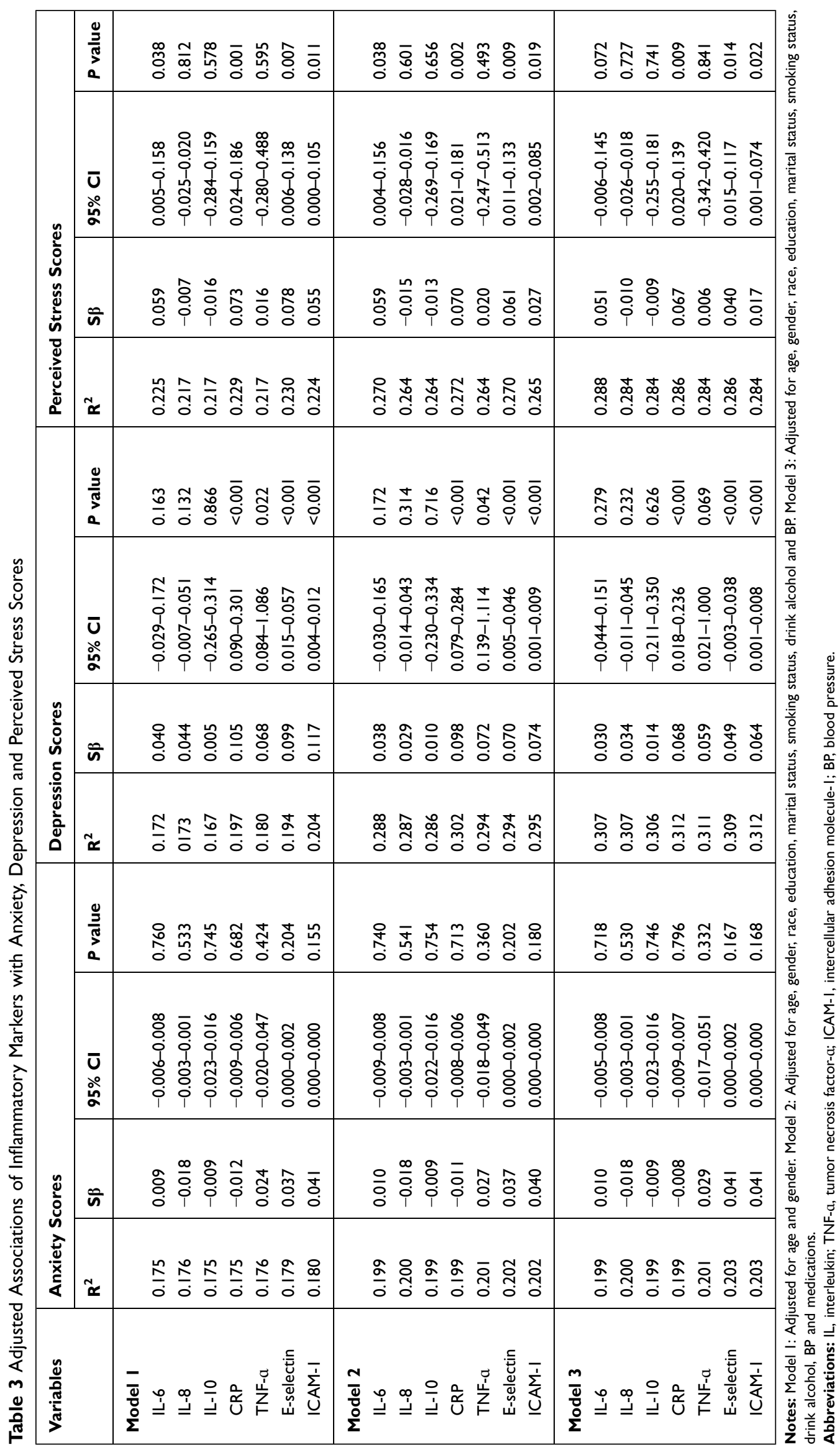




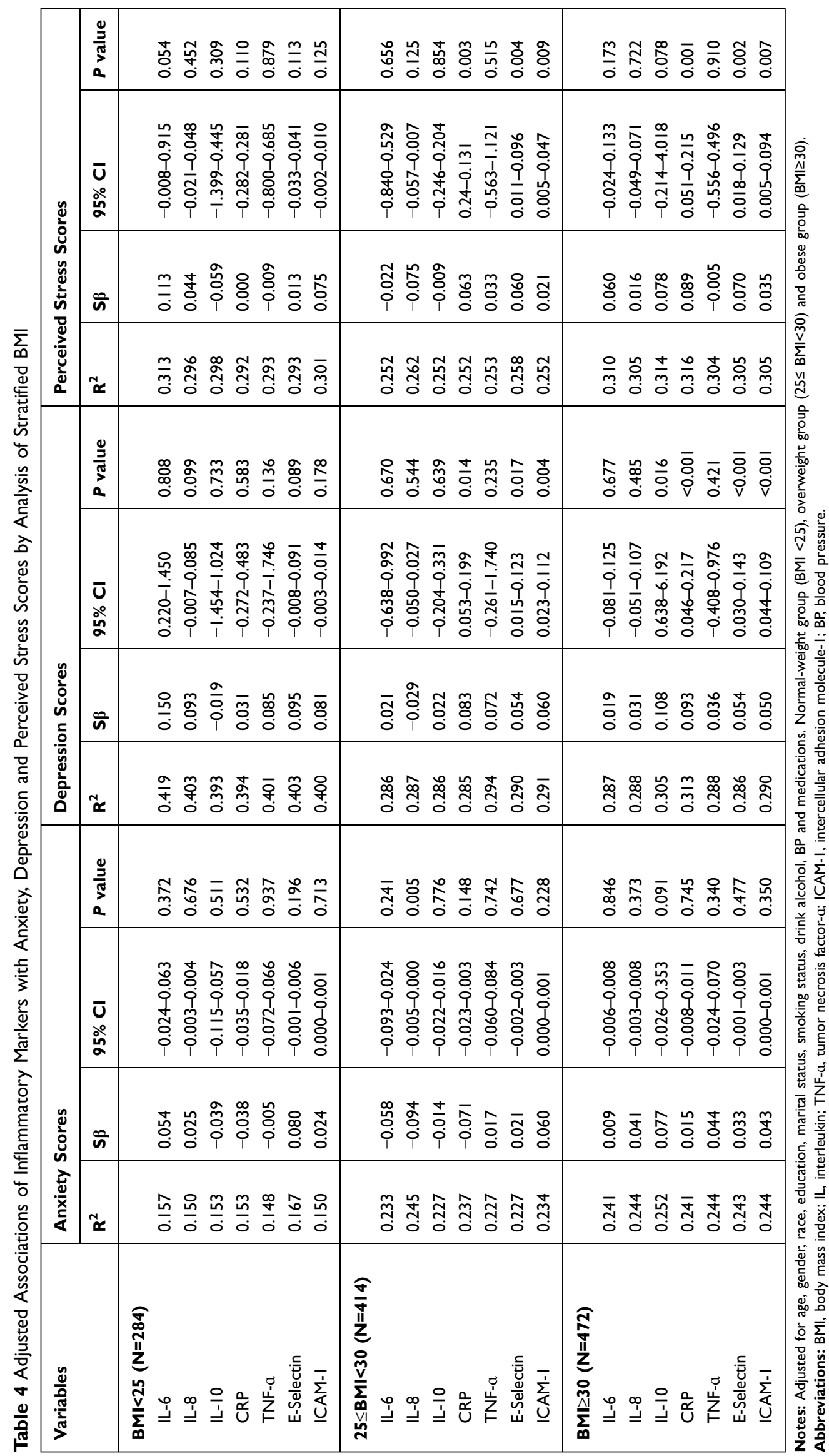


to analyze the relationship between inflammation and psychological scores, although none were associated with anxiety, depression and perceived stress.

Our results are consistent with many previous studies that reported a positive association between depressive symptoms and inflammation. ${ }^{33-36}$ However, several other studies found that depression has no or even an inverse correlation with inflammatory markers. ${ }^{37-39}$ These discrepancies may be due to the different study populations, study designs and confounding factors included. Based on previous research results, we further confirmed an association between depression and inflammatory markers independent of BMI in obese individuals. Our results support the hypothesis that the systemic inflammatory response may partially mediate a significant relationship between psychological scores and chronic inflammatory diseases such as CVDs in which inflammatory biomarkers provide a pathway linking depression and perceived stress to an increased risk of obesity-related CVDs in obese populations. ${ }^{1-9}$

Regarding anxiety, some studies have suggested a positive relationship between anxiety and serum levels of inflammatory markers. ${ }^{7-9,40}$ Previous studies reported that anxiety symptoms were associated with increased levels of TNF- $\alpha$, IL-6 and CRP. ${ }^{8,9,41}$ Duivis et al also observed elevated serum CRP levels in a male population after adjusting for sociodemographic and lifestyle-related confounding factors in a large adult sample. ${ }^{40}$ These previous results are inconsistent with our finding that the anxiety score was not associated with inflammation levels in a multivariate and stratified analysis. More comprehensive confounding factors, including sociodemographic characteristics, lifestyle, disease history and medications, were adjusted in our study, which may partly account for the differences in results. Additionally, distinct study designs, subject race, and blood test methods may account for the differences. $8,9,40$

Although many studies have suggested that perceived stress is closely related to chronic inflammatory diseases,22-25 studies on the association of perceived stress with inflammation levels were conflicting and few. ${ }^{42}$ To the best of our knowledge, this study is the first to show that the perceived stress score is significantly associated with inflammatory biomarkers such as CRP, E-selectin, and ICAM-1 among obese individuals, independent of sufficient and relevant confounding factors.

\section{Limitations}

First, all subjects were required to be healthy enough to travel to the clinical research centers; therefore, there is a possibility of population selection bias. Second, the causal relationship between the psychological scores and inflammation cannot be clarified in this cross-sectional study analysis. Third, the research population is mainly Caucasian. Whether the associations remain among other races warrants future investigation.

\section{Conclusions}

Our findings suggest that depression and perceived stress are independently and significantly linked with elevated serum levels of ICAM-1, E-selectin and CRP in overweight and obese populations. Chronic inflammatory activation caused by obesity may be amplified by depression and perceived stress, which can lead to obesity-related complications such as CVDs.

\section{Disclosure}

The authors report no conflicts of interest for this work.

\section{References}

1. Daneshzad E, Keshavarz S-A, Qorbani M, et al. Association between a low-carbohydrate diet and sleep status, depression, anxiety, and stress score. J Sci Food Agric. 2020;100(7):2946-2952. doi:10.1002/jsfa.10322

2. Huang Y, Su Y, Jiang Y, Zhu M. Sex differences in the associations between blood pressure and anxiety and depression scores in a middle-aged and elderly population: the Irish Longitudinal Study on Ageing (TILDA). J Affect Disord. 2020;274:118-125. doi:10.1016/j. jad.2020.05.133

3. Fiedorowicz JG, He J, Merikangas KR. The association between mood and anxiety disorders with vascular diseases and risk factors in a nationally representative sample. $J$ Psychosom Res. 2011;70 (2):0-154. doi:10.1016/j.jpsychores.2010.07.010

4. Roest AM, Martens EJ, de Jonge P, et al. Anxiety and risk of incident cardiovascular disease risk. J Am Coll Cardiol. 2010;56(1):38-46. doi:10.1016/j.jacc.2010.03.034

5. Liu T, Ma Y, Zhang R, et al. Resveratrol ameliorates estrogen deficiency-induced depression- and anxiety-like behaviors and hippocampal inflammation in mice. Psychopharmacology. 2019;236 (4):1385-1399. doi:10.1007/s00213-018-5148-5

6. Lamers F, Oppen PV, Comijs HC, et al. Comorbidity patterns of anxiety and depressive disorders in a large cohort study: the Netherlands Study of Depression and Anxiety (NESDA). J Clin Psychiatry. 2011;72(3):341-348. doi:10.4088/JCP.10m06176blu

7. Bankier B, Barajas J, Martinez-Rumayor A, et al. Association between C-reactive protein and generalized anxiety disorder in stable coronary heart disease patients. Eur Heart J. 2008;29(18):22122217. doi:10.1093/eurheartj/ehn326

8. Liukkonen T, Rasanen P, Jokelainen J, et al. The association between anxiety and C-reactive protein (CRP) levels: results from the Northern Finland 1966 birth cohort study. Eur Psychiatry. 2011;26 (6):363-369. doi:10.1016/j.eurpsy.2011.02.001

9. Duivis HE, Vogelzangs N, Kupper N, et al. Differential association of somatic and cognitive symptoms of depression and anxiety with inflammation: findings from the Netherlands Study of Depression and Anxiety (NESDA). Psychoneuroendocrinology. 2013;38 (9):1573-1585. doi:10.1016/j.psyneuen.2013.01.002 
10. Ridker PM. Clinical application of C-reactive protein for cardiovascular disease detection and prevention. Circulation. 2003;107 (3):363-369. doi:10.1161/01.CIR.0000053730.47739.3C

11. None. C-reactive protein, fibrinogen, and cardiovascular disease prediction. $N$ Engl J Med. 2012;367(14):1310-1320. doi:10.1056/ NEJMoa1107477

12. Choi J, Joseph L, Pilote L. Obesity and C-reactive protein in various populations: a systematic review and meta-analysis. Obes Rev. 2013;14(3):232-244. doi:10.1111/obr.12003

13. Cattane N, Rikknen K, Anniverno R, et al. Depression, obesity and their comorbidity during pregnancy: effects on the offspring's mental and physical health. Mol Psychiatry. 2020;1-20.

14. Jones-Corneille LR, Wadden TA, Sarwer DB, et al. Axis I psychopathology in bariatric surgery candidates with and without binge eating disorder: results of structured clinical interviews. Obes Surg. 2012;22(3):389-397. doi:10.1007/s11695-010-0322-9

15. Rosenberger PH, Henderson KE, Grilo CM. Psychiatric disorder comorbidity and association with eating disorders in bariatric surgery patients: a cross-sectional study using structured interview-based diagnosis. J Clin Psychiatry. 2006;67(7):1080-1085. doi:10.4088/ JCP.v67n0710

16. Katta R, Sanchez A, Tantry E. An anti-wrinkle diet: nutritional strategies to combat oxidation, inflammation and glycation. Skin Therapy Lett. 2020;25(2):3-7.

17. de Toda IM, Maté I, Vida C, et al. Immune function parameters as markers of biological age and predictors of longevity. Aging. 2016;8 (11):3110-3119. doi:10.18632/aging.101116

18. Salim S, Asghar M, Taneja M, et al. Potential contribution of oxidative stress and inflammation to anxiety and hypertension. Brain Res. 2011;1404:63-71. doi:10.1016/j.brainres.2011.06.024

19. Huang Y, Zhu M. Increased global PSQI score is associated with depressive symptoms in an adult population from the United States. Nat Sci Sleep. 2020;12:487-495. doi:10.2147/NSS.S256625

20. Fuente MDL, Gonzalez EM, Vida C. Increase of oxidation and inflammation in nervous and immune systems with aging and anxiety. Curr Pharm Des. 2014;20:29.

21. Vida C, de Toda IM, Cruces J, et al. Role of macrophages in agerelated oxidative stress and lipofuscin accumulation in mice. Redox Biol. 2017;12:423-437. doi:10.1016/j.redox.2017.03.005

22. Luigi F, Elisa F. Inflammageing: chronic inflammation in ageing, cardiovascular disease, and?Frailty. Nat Rev Cardiol. 2018;15 (9):505-522.

23. Koene RJ, Prizment AE, Blaes A, et al. Shared risk factors in cardiovascular disease and cancer. Circulation. 2016;133(11):1104 1114. doi:10.1161/CIRCULATIONAHA.115.020406

24. Noemí G, Cecilia Z, Leopoldo AA. Oxidative stress and inflammation in cardiovascular disease. Oxid Med Cell Longev. 2017;2017:1-2.

25. Tawakol A, Ishai A, Takx RA, et al. Relation between resting amygdalar activity and cardiovascular events: a longitudinal and cohort study. Lancet. 2017;389(10071):834-845. doi:10.1016/S0140-6736 (16)31714-7
26. Dienberg Love G, Seeman TE, Weinstein M, et al. Bioindicators in the MIDUS national study: protocol, measures, sample, and comparative context. J Aging Health. 2010;22(8):1059-1080. doi:10.1177/ 0898264310374355

27. Gruenewald TL, Karlamangla AS, Hu P, et al. History of socioeconomic disadvantage and allostatic load in later life. Soc Sci Med. 2012;74(1):75-83. doi:10.1016/j.socscimed.2011.09.037

28. Library WP. Hospital anxiety and depression scale[M]. 2010.

29. Radloff LS. The CES-D scale: a self-report depression scale for research in the general population. Appl Psychol Meas. 1977;1 (3):385-401. doi:10.1177/014662167700100306

30. Cohen S. A global measure of perceived stress. J Health Soc Behav. $1983 ; 24$.

31. Ghorpade DS, Ozcan L, Zheng Z, et al. Hepatocyte-secreted DPP4 in obesity promotes adipose inflammation and insulin resistance. Nature. 2018;555(7698):673-677. doi:10.1038/nature26138

32. Park EJ, Lee JH, Yu GY, et al. Dietary and genetic obesity promote liver inflammation and tumorigenesis by enhancing IL-6 and TNF expression. Cell. 2010;140(2):0-208. doi:10.1016/j.cell.2009.12.052

33. Jun Z, Yue Y, Thapa A et al. Baseline serum C-reactive protein levels may predict antidepressant treatment responses in patients with major depressive disorder. J Affect Disord. 2019.

34. Vetter ML, Wadden TA, Vinnard C, et al. Gender differences in the relationship between symptoms of depression and high-sensitivity CRP. Int J Obes. 2013;37(S1):S38-S43. doi:10.1038/ijo.2013.95

35. Elovainio M, Aalto AM, Kivim?Ki M, et al. Depression and C-reactive protein: population-based health 2000 study. Psychosom Med. 2009;71(4):423-430. doi:10.1097/PSY.0b013e31819e333a

36. Ford DE, Erlinger TP. Depression and C-reactive protein in US adults: data from the third national health and nutrition examination survey. Arch Intern Med. 2004;164(9):1010-1014. doi:10.1001/ archinte.164.9.1010

37. Ma J, Xiao L. Obesity and depression in US women: results from the 2005-2006 national health and nutritional examination survey. Obesity. 2009;18(2):347-353. doi:10.1038/oby.2009.213

38. Annique S, Dorien T, Richel L, et al. Inflammatory markers in depressed post-myocardial infarction patients. J Psychiatr Res. 2005;39(2):137-144. doi:10.1016/j.jpsychires.2004.05.009

39. Bremmer MA, Beekman ATF, Deeg DJH, et al. Inflammatory markers in late-life depression: results from a population-based study. $J$ Affect Disord. 2008;106(3):249-255. doi:10.1016/j.jad.2007.07.002

40. Black CN, Bot M, Scheffer PG, et al. Oxidative stress in major depressive and anxiety disorders, and the association with antidepressant use; results from a large adult cohort. Psychol Med. 2017;47 (5):936-948. doi:10.1017/S0033291716002828

41. Pierce GL, Kalil GZ, Ajibewa T, et al. Anxiety independently contributes to elevated inflammation in humans with obesity. Obesity. 2017;25(2):286-289. doi:10.1002/oby.21698

42. Huang Y, Jiang Y, Zhu M. The relationship between global sleep score and inflammatory markers in obese adults from the United States. Nat Sci Sleep. 2019;11:317-324. doi:10.2147/NSS.S220436
Risk Management and Healthcare Policy

\section{Publish your work in this journal}

Risk Management and Healthcare Policy is an international, peerreviewed, open access journal focusing on all aspects of public health, policy, and preventative measures to promote good health and improve morbidity and mortality in the population. The journal welcomes submitted papers covering original research, basic science, clinical \& epidemiological studies, reviews and evaluations, guidelines, expert opinion and commentary, case reports and extended reports. The manuscript management system is completely online and includes a very quick and fair peer-review system, which is all easy to use. Visit http://www.dovepress.com/testimonials.php to read real quotes from published authors. 